\title{
Extrapolations of nuclear binding energies from new linear mass relations
}

\author{
D. Hove, A.S. Jensen, K. Riisager \\ Department of Physics and Astronomy, Aarhus University, DK-8000 Aarhus C, Denmark
}

(Dated: February 28, 2022)

\begin{abstract}
We present a method to extrapolate nuclear binding energies from known values for neighbouring nuclei. We select four specific mass relations constructed to eliminate smooth variation of the binding energy as function nucleon numbers. The fast odd-even variations are avoided by comparing nuclei with same parity. The mass relations are first tested and shown to either be rather accurately obeyed or revealing signatures of quickly varying structures. Extrapolations are initially made for a nucleus by applying each of these relations. Very reliable estimates are then produced either by an average or by choosing the extrapolation where the smoothest structures enter. Corresponding mass relations for $Q_{\alpha}$ values are used to study the general structure of super-heavy elements. A minor neutron shell at $N=152$ is seen, but no sign of other shell structures are apparent in the super-heavy region. Accuracies are typically substantially better than $0.5 \mathrm{MeV}$.
\end{abstract}

PACS numbers: 21.10.Dr, 21.60.-n

\section{INTRODUCTION}

The importance of accurate knowledge of nuclear masses is not disputed by anybody. Unprecedented numbers of precise measurements are available [1, 2, but many particle stable masses are still unknown. The masses are collected in comprehensive mass tables [3] which also contain estimates based on smooth extra/interpolations and consistency between a number of related particle and cluster separation energies.

Different types of theoretical models are also used to estimate and predict nuclear masses of interest. They are almost all at some point employing phenomenological parametrization. The original example is the semiclassical mass formula by von Weizäcker and Bethe [4, where four parameters are fitted to known masses and all others can be predicted. Much more sophisticated versions are developed where the same idea of expanding in terms of neutron and proton numbers systematically is exploited in the liquid droplet model [5, 6].

The success of the liquid drop models is due to the overall continuous behaviour of nuclear masses as function of neutron and proton numbers, and of course on the inclusion of the correct physics ingredients of volume, surface, Coulomb and symmetry terms. After the bulk part of the nuclear masses are described the smaller contributions are highlighted as the remaining part. This is much more difficult to describe as the origin is in a number of very different correlations expressed as e.g. shell effects, deformations, and pairing. These three correlations occur rather systematically and can to some degree be accounted for in the droplet models. However, the severe limitation is that predictions beyond the experimentally known regions quickly become rather inaccurate.

Improvement in predictive power is obtained by microscopic mean-field models, i.e. Hartee-Fock-Boguliubov, Density Functional Theory and Thomas-Fermi calculations [7, 8. Now the phenomenology enters as the nucleon-nucleon interactions used as input, and determined from general symmetry principles and by fitting to resulting computed properties. Here the self-consistency is necessary to have reliability beyond the fitted regions. At some level the liquid drop bulk properties must be reproduced if these models are to be successful. This is more directly exploited in the micro-macro models where the microscopic fluctuating part first is extracted from a mean-field shell model computation and the average smooth part is replaced by liquid drop expressions [9].

The origin of nuclear masses is the nucleon-nucleon interaction which implies that the different nuclei have (perhaps complicated) related masses. This is explored in ab initio calculations of nuclear masses from the basic interaction [10]. It is exploited in a completely different way in a series of so-called mass relations where GarveyKelson is the most well-known [11. It is based on counting the number of pairwise interactions in different nuclei and by adding for example three mass difference between two nuclei, the result should be zero. This is tested to be true for known masses with an average accuracy of about $500 \mathrm{keV}$ [12].

It is then interesting to test whether the previous mass formulae obey the rather accurate Garvey-Kelson mass relations. This turns out to be essentially true for the measured masses, but as soon as extrapolations are involved the accuracy drops by about a factor of two 13 . The phenomenology is only really trustworthy within the fitted region. There is apparently one exception in the Duflo-Zuker mass formulae constructed from the same principles as the Garvey-Kelson mass relations [13, 14.

A different principle was used in extraction of pairing properties where emphasis rather than cancellation is desired. Odd-even mass differences between neighbouring nuclei already reveal these effects. An improvement is obtained in the slightly more complicated combination where an average of the two neighbouring mass differences is used 4. A further extension to include more masses led Jensen et al. [15] to formulate mass relations obeying a general principle of cancellation of all smooth terms up to any desired order. The practical choice is second order, since the necessary nuclei otherwise may differ 
too much. It is interesting to note that Garvey-Kelson relations also eliminate all smooth terms up to second order. Although never emphasized previously, this is obviously a convincing reason for their success.

Different mass combinations can now be chosen to either emphasize specific correlations or to avoid them for example by cancellation. The latter choice produces a combination of masses equal to zero, which means any of these masses can be expressed as a linear combination of the other ones. Thus, if correct such mass relations are directly applicable for one-step extrapolations beyond known mass regions. Similar extrapolations can be made with Garvey-Kelson mass relations but they do not allow special choices where for example odd-even effects a priori are absent or emphasized. Other correlations could be investigated as well if a mass combination can be found to highlight them.

It has been suggested that nuclear masses have a component of chaotic behaviour amounting to $2.78 / A^{1 / 3} \mathrm{MeV}$ [16] which amounts to between 0.5 and $1.4 \mathrm{MeV}$. This seems to be an exaggeration as suggested by the observation that specific regions exhibit (unknown) correlations 17 accounting for maybe half of this amount. This is also indicated by the rather small root mean square deviation of less than $100 \mathrm{keV}$ obtained by the 12 point Garvey-Kelson mass interpolation [13. Thus, any mass extrapolation can ultimately only meaningfully aim for an accuracy of at most $200-300 \mathrm{keV}$ with global mass formulae.

The purpose of this paper is to present four linear mass relations between isotopes capable of removing smoothly varying contributions. When applied to isotopes with measured binding energies, these mass combinations should have a tendency to cancel completely barring influences from other significant contributing factors. Expressing unknown masses as linear combinations of known ones should allow for the extrapolating of these unknown masses. This will all be based on isotopes in their ground state configurations. The relatively few assumptions needed to establish the fundamental model is the greatest advantage of the method. As a result all conclusions will be based purely on combinations of binding energies, without the need for other theoretical considerations.

Our focus will be divided between extrapolating unknown binding energies and studying the structure of the super-heavy elements. We shall use the method introduced in [15] to construct mass relations. Here it will not be attempted to verify the existence and scale of the effects that influence the binding energy. We shall use suitable mass relations to eliminate all or most of the systematic dependencies of the binding energy on nucleon numbers. The legitimacy of the elimination will be apparent from the results of applying the mass relations.

The fundamental model, along with the argumentation supporting it, will be presented in sect. (III). The majority of the necessary formulations will be included there as well. It is then possible to define the specific mass relations needed for the applications, and this is also included in sect. (III).

Applying these mass relations individually with the purpose of extrapolating to new binding energies is done in sect. (III A) and (IIIB). In sect. (III C) the mass relations are used with $Q_{\alpha}$ values. This has a number of advantages. In particular, it is possible to examine the region of super-heavy isotopes in greater detail. The $Q_{\alpha}$ values are very useful in analysis of general structures appearing in the binding energy. By comparing extrapolations from the individual mass relations it is possible to calculate more precise results either by simple averages or by choosing the smoothest extrapolation. Such combinations are presented in sect. (IV) along with the numerical results in table $\mathrm{I}$. Finally, sect. (V) contains a brief summary and the conclusions.

\section{THE MASS RELATIONS}

The idea behind the mass relations is that the nuclear many-body systems all are derived from the same basic interactions, and hence different nuclei should have related binding energies. Various principles are applied for different mass relations. We shall focus on one type where we first describe the general principles, then we derive some useful properties, and finally we specify the applications in the last subsection.

\section{A. General assumptions}

The mass formula is often divided into a sum of three different types of terms. First the dominating term, $B_{L D}(N, Z)$, describing the smoothly varying gross properties of the binding energy as function of neutron and proton numbers, $N$ and $Z$. This is the liquid drop, or droplet, model with the classical four terms, that is volume-, surface-, Coulomb- and symmetry-energy. The specific form and the precise numerical values are not important since the smooth character is only necessary to eliminate unwanted contributions. This is achieved through suitable linear combinations of the nuclear binding energies as elaborated in sect. (IIB).

Second, a term accounting for shell effects, $B_{s h}(N, Z)$, arising from quantum mechanical correlations favouring special (spherical) configurations. Third, a term, $\Delta(N, Z)$, describing systematic but not smoothly varying contributions to the binding energy. This can be oddeven and other similar (short-ranged) correlation effects. In total, we have the binding energy separated into such distinct terms, where each is a function of the nucleon numbers $N$, and $Z$ :

$$
B(N, Z)=B_{L D}(N, Z)+B_{s h}(N, Z)+\Delta(N, Z) .
$$

Explicit addition of terms describing other effects, for instance the possible tendency to form $\alpha$-particles within 
nuclei, could also be included. However, the possible nature of $\alpha$-clusters is presently not our prime focus, and furthermore the energy gain from these clusters are also very small or possibly very smoothly varying [15.

Since the existence of both neutron and proton shells is undeniable, the second term, $B_{s h}(N, Z)$, is an inescapable necessity. The major shells are prominent only in relatively narrow regions of nucleon numbers. A slowly varying contribution from $B_{s h}$ between shells can then essentially be eliminated by the same procedure as $B_{L D}(N, Z)$. This claim will be substantiated by the results in sect. (III).

The systematic third term, $\Delta(N, Z)$, is more complicated since it is composed of several effects. It includes three different pairing effects, along with the Wigner term related to the isospin symmetry, all of which are more subtle in nature than the smooth term. However, they are all smooth functions of nucleon numbers provided isotopes with same parity are compared and the $N=Z$ line is not crossed. We shall in this paper impose these restrictions on the employed extrapolations, although we expect to encounter occasional signals of these terms.

The terms in eq. (1) do not necessarily constitute a complete expression for the binding energy. Additional overlooked or unknown effects might also contribute in different ways. However, we expect that any such neglected but significant effects will produce a clear deviation from the systematic results, and thereby reveal itself. This will be considered in relation to the actual numerical results presented in sect. (IV).

\section{B. Manipulating the binding energy}

A flexible method to manipulate binding energies was discussed in [15] with the aim of isolating specific contributing effects. A possibility is then to study individual effects in relative isolation. However, this flexibility also indirectly enables the extrapolation of unknown binding energies. The idea is to combine separation energies in a manner reminiscent of a second order difference.

$$
\begin{aligned}
Q\left(n_{1}, z_{1} ; n_{2}, z_{2}\right)= & -S\left(N-n_{1}, Z-z_{1}\right)+2 S(N, Z) \\
& -S\left(N+n_{1}, Z+z_{1}\right) .
\end{aligned}
$$

The separation energy of $n_{2}$ neutrons and $z_{2}$ protons in any isotope is given as a difference between binding energies.

$$
S(N, Z)=B(N, Z)-B\left(N-n_{2}, Z-z_{2}\right) .
$$

Calculating $Q$ using eqs. (1) and (3) results in an expression for $Q$, which like the original expression for $B$ in eq. (1), can be separated in three terms, i.e.

$$
Q=Q_{L D}+Q_{s h}+Q_{\Delta} .
$$

Depending on the chosen $\left(n_{1}, z_{1} ; n_{2}, z_{2}\right)$, some terms will be diminished while others will be emphasized. The contributions from the last two terms in eq. (4) vary greatly in size depending on the chosen $\left(n_{1}, z_{1} ; n_{2}, z_{2}\right)$, but common for all configurations is the fact that the smooth terms are almost completely eliminated. Interpreting the discrete variables $N$ and $Z$ as global, continuous variables automatically results in an elimination up to and including the second order in the Taylor expansion of the smooth terms around $(N, Z)$. The leading order contribution to continuous functions, $\tilde{B}$ and $\tilde{Q}$ analogous to $B$ and $Q$, is then third order in the Taylor expansion, that is

$$
\begin{aligned}
\tilde{Q}= & -\frac{\partial^{3} \tilde{B}}{\partial N^{2} \partial Z} n_{1}\left(n_{1} z_{2}+2 n_{2} z_{1}\right)-\frac{\partial^{3} \tilde{B}}{\partial N^{3}} n_{1}^{2} n_{2} \\
& -\frac{\partial^{3} \tilde{B}}{\partial Z^{3}} z_{1}^{2} z_{2}-\frac{\partial^{3} \tilde{B}}{\partial N \partial Z^{2}} z_{1}\left(n_{2} z_{1}+2 n_{1} z_{2}\right),
\end{aligned}
$$

as seen by direct expansion. This remaining contribution will always be present for smooth functions when mass relations based on eq. (2) are constructed. It either has to be corrected for or included in accuracy estimates.

By severe reduction of the smooth contributions to a size like in eq. (5) other effects would stand out. Desired effects can then be emphasized by suitably chosen configurations $\left(n_{1}, z_{1} ; n_{2}, z_{2}\right)$. The shell effect in particular will figure prominently in certain parts of the nuclear chart, and the validity of some extrapolations in these areas will therefore be more doubtful. However, lacking an accurate expression for the general contributions from shell effects, it is difficult to construct appropriately corrected mass relations. Also any expression describing shell effects would be another source of error in the extrapolations. Thus, we shall not attempt to account for the shell effects, although perhaps detect their presence by observing systematic deviations.

Instead of the separation energies in eq. (2) we can use similar combinations arising from $Q_{\alpha}$ values, that is for $n_{2}=z_{2}=2$

$$
S(N, Z)-B\left({ }^{4} \mathrm{He}\right)=-Q_{\alpha} .
$$

The advantage is that $Q_{\alpha}$ sometimes is much more accurately known than nuclear masses themselves, and this is especially pronounced for super-heavy nuclei. This observation is very well established by the experimental techniques where masses are measured relative to other masses. Then it is possible to use eq. (2) with $Q_{\alpha}$ values, which leads to

$$
\begin{aligned}
Q= & Q+B\left({ }^{4} \mathrm{He}\right)-2 B\left({ }^{4} \mathrm{He}\right)+B\left({ }^{4} \mathrm{He}\right) \\
= & Q_{\alpha}\left(N-n_{1}, Z-z_{1}\right)-2 Q_{\alpha}(N, Z) \\
& +Q_{\alpha}\left(N+n_{1}, Z+z_{1}\right) .
\end{aligned}
$$

Other types of conclusions may then become possible from $Q_{\alpha}$ relations, as, in addition to the better accuracy, only three measured values enters eq. $(7)$ in contrast to the four terms arising from eqs. (2) and (3). 


\section{Constructing specific mass relations}

The aim is to find a reliable extrapolation of binding energies through the mass relations in sect. (II B). This is accomplished by carefully choosing the configuration in eq. (2) such that the result ideally is zero. If in a certain area of the nuclear chart, limited only by the available measured isotopes, a mass relation is prone to return the value zero, it is reasonable to assume this tendency would continue beyond the known isotopes. Unknown binding energies can then be calculated directly from a given mass relation. However, such extrapolation is only reliable if the chosen mass relation in fact eliminates all contributions from the binding energy in eq. (1). Even then care has to be taken to avoid outlandish results.

Many mass formulas have a tendency to deviate significantly when extrapolating outside the experimentally known region 13 . The present method does not rely on a specific form of a mass formula. However, eq. (2) allows for an endless number of possible mass relations by choosing $\left(n_{1}, z_{1} ; n_{2}, z_{2}\right)$ accordingly, and using too great values for $n_{i}$ and $z_{i}$ would make the approximation of eq. (2) as a derivative less accurate. The likelihood of combining different effects in the result increases when combining isotopes farther apart, and the extrapolation would also be less accurate. Similarly, the mass relation could be chosen to eliminate the smooth parts to any order desired. Unfortunately, this would also come at the expense of reliability since isotopes farther apart would be required.

We therefore only apply mass relations where $n_{i}$ and $z_{i}$ never are larger than 2. Furthermore, to avoid the quickly varying pairing contribution, we choose to compare nuclei of the same odd-even character. In total we use here four mass relations where $n_{i}$ and $z_{i}$ are 0 and 2 . They combine nuclei with fixed $N, Z, A=N+Z$, and $N-Z$, respectively, that is defined by

$$
\begin{aligned}
\Delta_{2 n}(N, Z) & =Q(2,0 ; 2,0) \\
\Delta_{2 p}(N, Z) & =Q(0,2 ; 0,2) \\
\Delta_{2 \alpha}(N, Z) & =Q(2,2 ; 2,2) \\
\Delta_{2(N-Z)}(N, Z) & =Q(2,-2 ; 2,-2) .
\end{aligned}
$$

The actual nuclei in these four mass relations can be seen in fig. 1 where the original six nuclei from eq. (2) and (3) reduce to only four with different weights. These four mass relations should, ideally, completely eliminate any contributions from pairing effects. Of course, the actual results will not be so idealized, and will at the very least include remnants of the smooth term. Some minor pairing contribution might still remain, since no systematic theory can account for all these effects as discussed by Friedman et al. [18]. Still, the combinations shown in fig. 1 seem intuitively to be more likely to add up to zero, and thereby providing useful mass relations for the extrapolations.

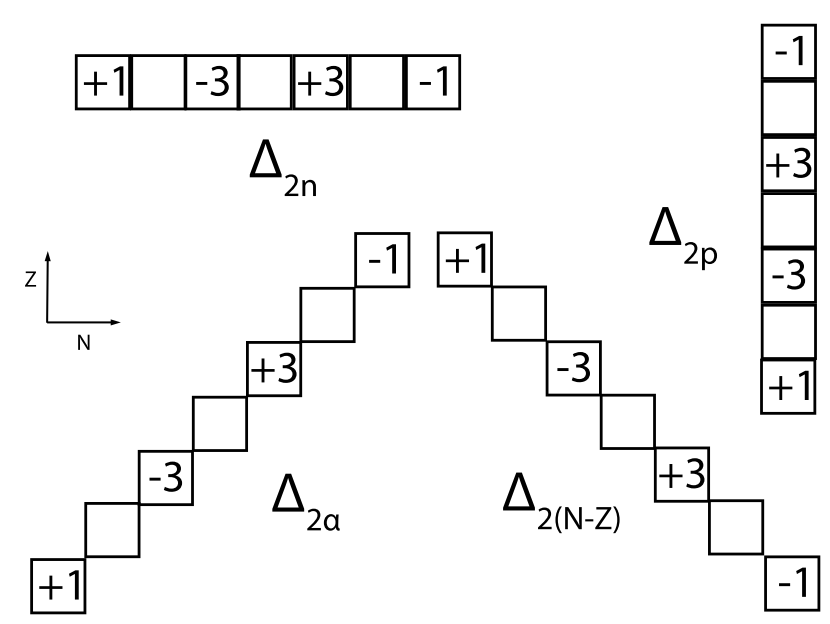

Figure 1. The physical structures of the four mass relations in eq. (8). The weights assigned to the different isotopes reflect the concatenation of the six terms from eqs. (2) and (3) into four.

\section{EXTRAPOLATIONS}

The actual analysis is divided into two subsections. First the results are examined individually from applying the four mass relations to the available measurements of binding energies. The tendencies are discussed to emphasize the relevant structures and provide insight into the viability of the general method. The areas accessible to the mass relations will also be determined in the process. The measurements are from Audi and Meng [21] for isotopes in their ground state with nucleon number, $A$, spanning $0-295$.

Second, the mass relations are applied to $Q_{\alpha}$ values with the purpose of analysing general structures found among the super-heavy elements. The measurements of $Q_{\alpha}$ are also from Audi and Meng [21, unfortunately, they are not necessarily of isotopes in their ground state.

\section{A. Procedure and general behavior}

The general method described in sect. (IIC) is idealized, and constitutes the simplest and most obvious way to perform the extrapolations. However, a slightly more complicated procedure is applied to increase accuracy and estimate uncertainty. The fundamental idea is still to combine four different isotopes either horizontally, vertically, or diagonally.

First the mass relation is tested locally, that is with $\Delta_{2 n}$ as the example and $(N+2, Z)$ as the unknown we compute $\Delta_{2 n}(N-2, Z), \Delta_{2 n}(N-4, Z)$, and $\Delta_{2 n}(N-$ $6, Z)$. Each would be zero if the mass relation is exactly obeyed. A systematic tendency in the region can be accounted for by computing the non-zero average value which is used for $\Delta_{2 n}(N, Z)$ in the prediction of the 
unknown $(N+2, Z)$ binding energy. Obviously, a systematic tendency is then accounted for in the prediction which furthermore now has an extrapolation uncertainty attached from the spread around the average value of the mass relation.

With the diagonal relations $\left(\Delta_{2(N-Z)}\right.$ and $\left.\Delta_{2 \alpha}\right)$ it is impossible to calculate an average based on three preceding values. This would reduce the available extrapolations almost to none. Instead only the immediately preceding value is used for $\Delta_{2(N-Z)}$.

The uncertainties of the actual extrapolations have two general sources. The uncertainties in the measurements combine with the uncertainty of the predicted (non-zero) average value. Since this expected average is based on three different, but overlapping applications of the mass relation, this statistical error is the combination of six different uncertainties in measurements. Depending on the specific isotopes, and how well they have been measured, this uncertainty can at times be very significant.

Recently, it has been suggested by Olofsson et al. [17. that the distribution of binding energies inherently is, at least partly, chaotic in nature. This is still subject to discussion as Molinari and Weidenmüller [16] interpret the results as being due to residual interactions in the shell model. However, to account for any (chaotic) fluctuations the variation of the average value, computed from the three mass combinations, must be included in the final uncertainty of the extrapolated value.

To achieve this we combine the two different contributions to the uncertainty, that is from measurement and average. Thus, $r_{i} \pm \sqrt{s_{i}^{2}+v_{i}^{2}}=r_{i} \pm \sigma_{i}$, where $i$ labels the applied relation, $r_{i}$ is the extrapolated value, $s_{i}$ is the measurement uncertainty, $v_{i}$ is the variation, and $\sigma_{i}$ is the final uncertainty of the extrapolation. The applicability of this extrapolation method has limits, and some energies cannot be meaningfully extrapolated. Consequently, only results where $\sigma<500 \mathrm{keV}$ are included, since otherwise the extrapolated values are too uncertain to be of interest.

We now proceed to investigate the systematic behaviour of the mass relations. The results from all four mass relations are shown in figs. 2 and 3 The most prominent visible features arise from the shell effects around the more or less magic numbers. Whenever a shell crossing is involved, a significant deviation from the surrounding binding energies appear. How the mass relation is positioned relative to the shell defines both the sign and the scale of this deviation.

Consequently, because the binding is amplified by the +3 coefficient on the $(N, Z)$ value (see fig. 1), a mass relation computed for a magic number $(N, Z)$ must be significantly greater than for neighbouring isotopes. A mass relation centred one or possibly even two nucleons before a shell should also have a noticeably greater outcome, though not to the same extent. Similarly, a relation centred two or three nucleons after a shell would have a noticeably smaller outcome, since the -3 coefficient in the relation would be closer to the shell and would therefore dominate over the +3 contribution. If the relation was centred just after a magic number the -3 and +3 coefficients would probably cancel, and the result might appear as if unaffected by shells altogether.

Generally, it is tempting to assume that the extrapolations will be more exact in regions with heavier isotopes, where changes from isotope to isotope are more gradual. If the changes are more gradual, the expected outcome should presumably be more reliable, as the binding energies themselves would fluctuate less. This can also be reflected in the attached uncertainties.

\section{B. Results from individual mass relations}

The $\Delta_{2 n}$ and $\Delta_{2 p}$ relations are shown in fig. 2. They only combine nuclear masses horizontally and vertically in the $N-Z$ diagram. They are therefore well suited for extrapolations beyond neutron and proton drip lines, but less well suited for the narrow strips of super-heavy elements. These two mass relations are also only sensitive to their own type of shell effects as seen in the figures. This confirms again the almost independence of neutron and proton shell fillings. The very light isotopes have been omitted, because they disrupted the energy scale and made minor energy changes less obvious. Their binding energies and structure are in any case strongly varying and any meaningful extrapolation would be close to impossible.

The most prominent features in the $\Delta_{2 p}$ relation on fig. 2 are the shells at $Z=50$, and 82 , but also the shell at $Z=28$ is clearly visible. The trace of these shells extend over numbers corresponding to the range of the mass relations. As expected the influence is positive below and negative above the shells. This symmetry extends to both sides of a shell, and is reflected in the size as well. The absolute values at the shells vary, but is always greater than $1 \mathrm{MeV}$ and often $\sim 2-4 \mathrm{MeV}$. The results for $(N, Z)$ and $(N, Z-2)$, when located at the shell, are nearly identical with opposite sign, which again demonstrates the symmetry of the shell effect.

It is also interesting to note how neutron shells only are visible with $\Delta_{2 p}$ at a proton shell, otherwise the mass relation is very small. This emphasizes how exclusively $\Delta_{2 p}$ is concerned with effects relating to protons. The neutron and proton shells are to a large extent, away from drip lines, filled independently. The region around $Z=40$ where $N>50$, shows many characteristics otherwise found in shells. There is an increase in energy just before $Z=40$ and a decrease in energy afterwards, with a slight fluctuation at $Z=40$, which is similar to the shell at $Z=82$. The energy changes are less pronounced than for other shells and the energy changes are also less well-defined. Nevertheless, the general smooth behaviour in the region is clearly disrupted, and the result is compatible with $Z=40$ as the most prominent subshell.

Overall, the $\Delta_{2 p}$ relation has away from shells, a very 

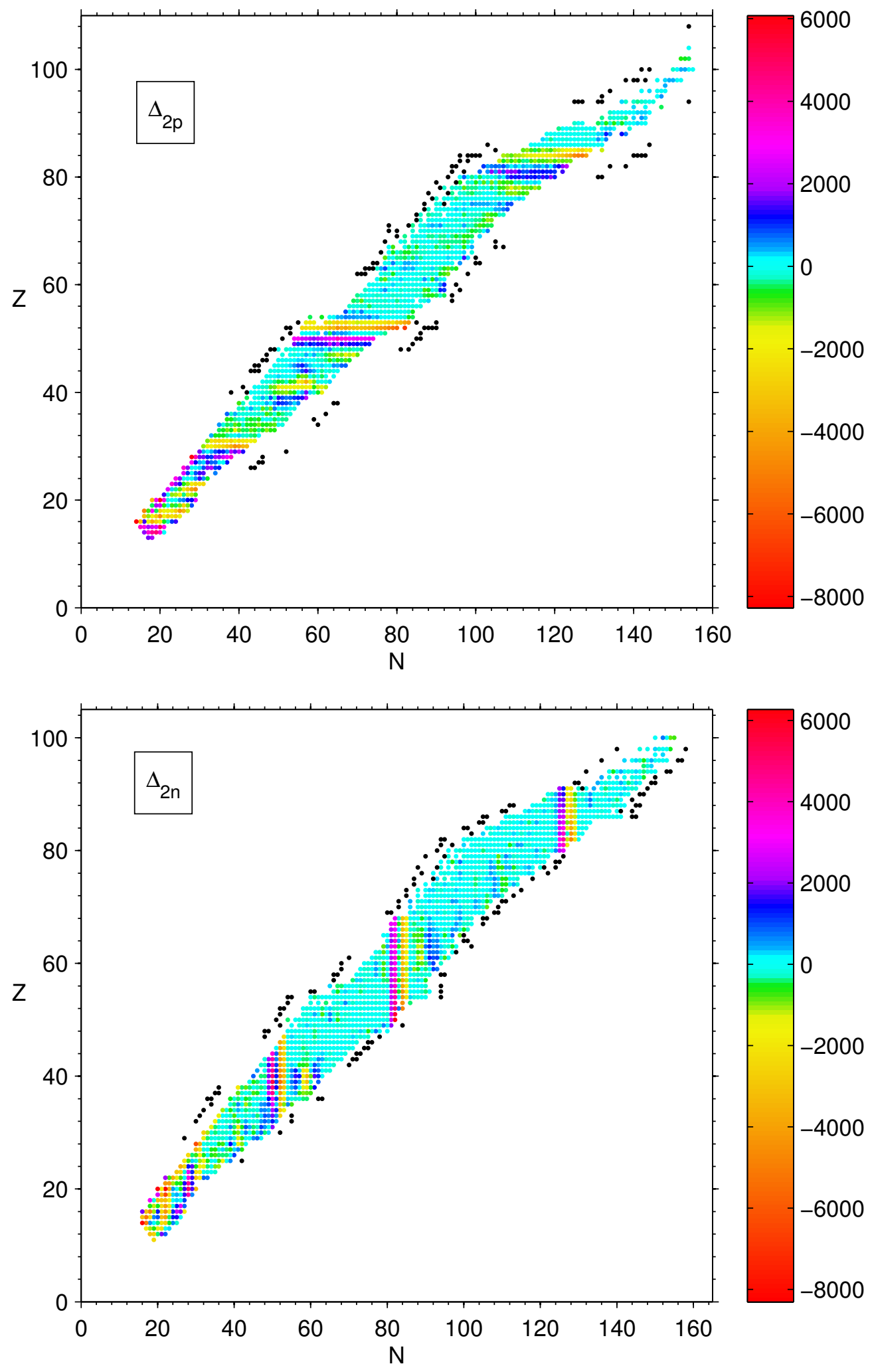

Figure 2. The vertical relation $\Delta_{2 p}$ with the horizontal relation $\Delta_{2 n}$ below applied to all isotopes with $A>30$. The colour scale is in $\mathrm{keV}$ and extrapolated isotopes are in black. 

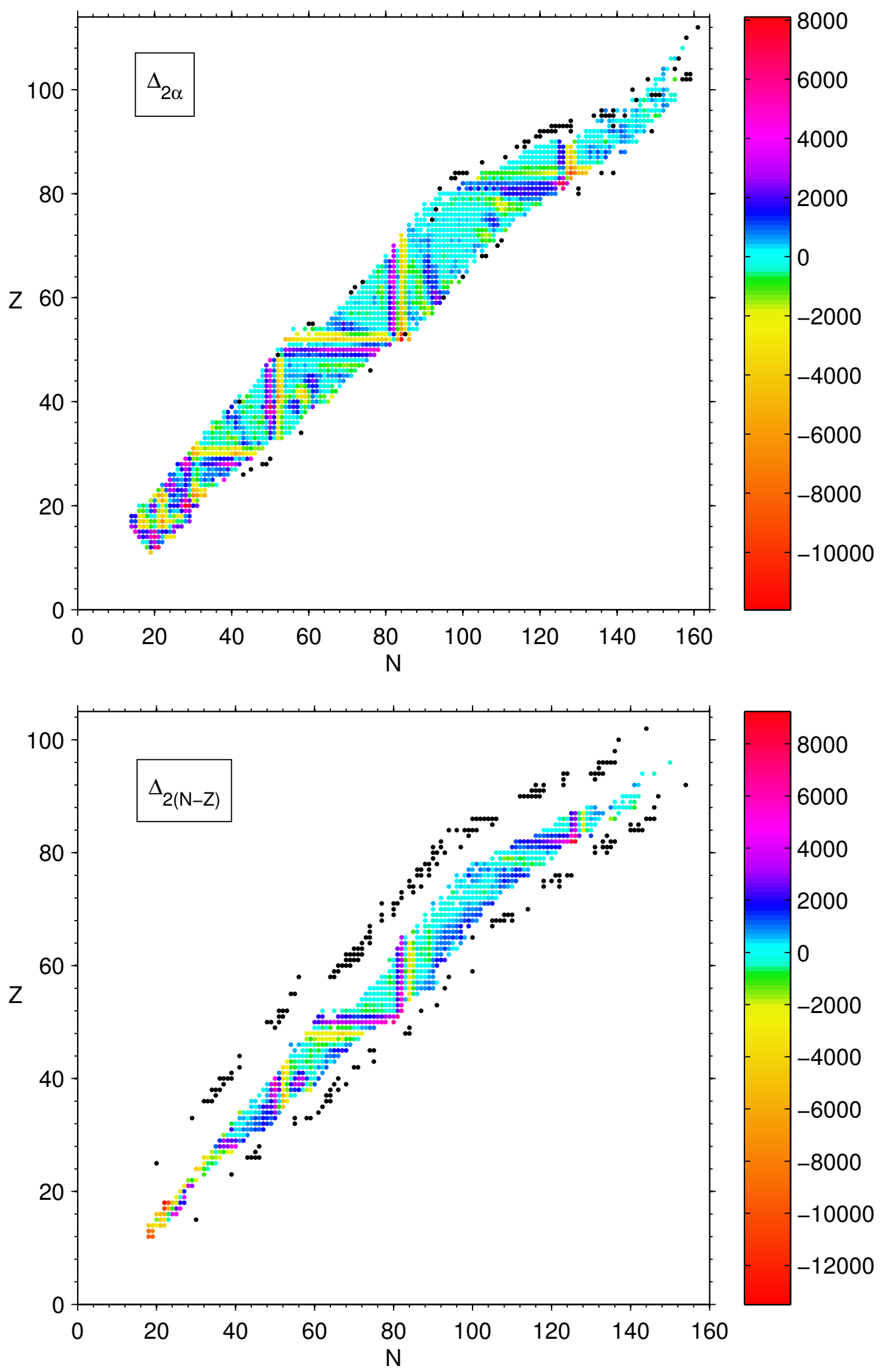

Figure 3. The two diagonal relations, $\Delta_{2 \alpha}$ above and $\Delta_{2(N-Z)}$ below applied to all isotopes with $A>30$. The colour scale is in $\mathrm{keV}$ and extrapolated isotopes are in black. 
pronounced tendency to more or less vanish. In particular, the region beyond the $Z=50$ shell is smooth and typically less than $500 \mathrm{keV}$ numerically. Extrapolations from this region should then be very reliable. This claim will be carefully investigated in sect. $(\overline{I V})$, where we also compare to extrapolations from other mass relations.

The results in fig. 2 from $\Delta_{2 n}$ are incredibly similar in most regards to the results for $\Delta_{2 p}$. The same tendency to complete cancellation is observed, though the remains are typically less than $300 \mathrm{keV}$ when evaluated numerically. Actually, every visible feature appears more distinctly. The shells at $N=28,50,82$, and 126 are not only obvious, they are sharply defined and confined to the area immediately surrounding the shells. The symmetry around the shell itself is also still present, and it is as clear as for protons. The size of the shell deviations are $\sim 2-4 \mathrm{MeV}$, again very much comparable to the $\Delta_{2 p}$ results.

More interesting is the region around $(Z, N)=$ $(40,60)$, where once again a deviation is visible. The same region where the $Z=40$ subshell was visible with $\Delta_{2 p}$ now shows a deviation with $\Delta_{2 n}$. This is particularly interesting considering that none of the major proton shells are visible away from a neutron shell, which suggest that this is not solely a shell effect. The deviation has some similarities with the other shells, but it is still decisively different from an ordinary shell. Most strikingly, the energy first increases, then decreases, and then increases again, which again suggests that this effect arises from a more complicated structure than a regular shell effect.

As an example of the possible use of these mass relations we look into this mass region in a little more details. The figs. $2 \mathrm{a}$ and $2 \mathrm{~b}$ clearly show shell structures around $(Z, N)=(40,58)$. First, the neutron shell at $N=58$ is less prominent than the well established major shells, but nevertheless unmistakingly recognized by the mass relations deviating from zero. This observation of a neutron subshell for $N=58,60$ is discussed in [19].

These shells for $Z=40$ and $N=58$ do not extend through all the known isotopes. For $Z=40$, the structure is absent for $N<49$, and present for $49<N<63$. For $N=58$, the structure is absent for $Z>42$, and present for $38<Z<42$. The explanations can be found by inspecting the fillings of the corresponding neutron and proton shells. For nucleon numbers between 40 and 50 , the $g_{9 / 2}$ shell is only partly occupied. Adding more nucleons require occupation of other shells, that is $g_{7 / 2}, d_{5 / 2}$ and possibly $s_{1 / 2}$. Then the neutron shell at $N=58$ disappears when $Z$ increases beyond 42 . This is precisely when at least 4 protons occupy the $g_{9 / 2}$ shell which therefore wants to deform to avoid the degeneracy. The neutron shell is not sufficiently strong to prevent this deformation. For $Z=40$, the proton shell is only visible for $N$ larger than 48, which is when the rather close-lying $g_{7 / 2}$ and $d_{5 / 2}$ levels begin to be occupied. The gain in neutron deformation energy is not sufficient to overcome the rather strong spherical proton shell effect. The rea- son is that the neutron single-particle level density only changes relatively little with modest deformation.

The results from using the diagonal relations for $\Delta_{2 \alpha}$ and $\Delta_{2(N-Z)}$ are presented in fig. 3 The $\Delta_{2 \alpha}$ relation is oriented diagonally towards the heavy isotopes in the chart of nuclides. It should therefore be able to extrapolate to heavier isotopes than any of the other mass relation. Unfortunately, this orientation also rather strongly confines it to the isotopes at the heavy end of known isotopes.

The shells are again very pronounced, but now all the structures from both $\Delta_{2 p}$ and $\Delta_{2 n}$ appear in $\Delta_{2 \alpha}$. It is interesting to notice how the neutron shells are more sharply defined than the proton shells, as it also appeared when comparing the results of $\Delta_{2 p}$ and $\Delta_{2 n}$. Not surprisingly, the deviation around $(Z, N)=(40,60)$ is even more prominent here, but also the area around $(Z, N)=(60,92)$ shows a rather strong deviation from zero. This deviation could also be detected with $\Delta_{2 n}$, albeit more faintly, but it was almost invisible with $\Delta_{2 p}$. A clear and significant effect in this mass region is therefore somewhat surprising, but it demonstrates how well $\Delta_{2 \alpha}$ detects the more elusive tendencies.

As $\Delta_{2 \alpha}$ includes more effects than $\Delta_{2 p}$ and $\Delta_{2 n}$, the results also vary much more. The reliability of any extrapolated result might therefore be questionable. This objection is legitimate for extrapolations involving several different shells. However, away from shells the fluctuations around zero are generally less than $500 \mathrm{keV}$. In particular, a promising mass region with smooth behaviour is $N>126$ and $Z>82$. For heavy or super-heavy isotopes the results should be as reliable as with $\Delta_{2 p}$ or $\Delta_{2 n}$ at the drip lines. This suggests interesting extrapolations with $\Delta_{2 \alpha}$ in the less accessible region of heavy or super-heavy nuclei.

Finally, the other diagonal relation, $\Delta_{2(N-Z)}$, is shown in fig. (3). Unfortunately, it is oriented perpendicular to the rather narrow strip of measured masses. The number of isotopes to which this relation can be readily applied is therefore rather limited. On the other hand it points directly towards the boundary of the known nuclear territory which then should allow extrapolations coinciding with $\Delta_{2 n}$ and $\Delta_{2 p}$. However, the general behaviour is the same as with $\Delta_{2 \alpha}$, and both proton and neutron shells are clearly visible. This limits the possibilities for reliable extrapolations.

These discussions suggest that the mass relation with the largest extrapolation potential seem to be the $\Delta_{2 n}$ relation. It generally cancels completely, is affected by few unpredictable effects, and it sharply defines the neutron shells. The same is true for the $\Delta_{2 p}$ relation, although its nature is slightly more erratic. The diagonal relations $\Delta_{2(N-Z)}$ and $\Delta_{2 \alpha}$ should be as reliable as $\Delta_{2 n}$ or $\Delta_{2 p}$, but more care must be taken when applying them, as they are more often influenced by shells.

Viewed collectively the results unanimously corroborate the predictions from sect. (II), which in turn indicates that the initial division of the binding energy in the 
three characteristic terms is well founded.

\section{Evaluations with $\mathbf{Q}_{\alpha}$ values}

The available measurements of $Q_{\alpha}$ values extend to far heavier isotopes than the binding energies, although recent developments at SHIPTRAP [20] in measuring absolute masses may allow for more extensive use of our method in the future. $Q_{\alpha}$ can therefore provide greater insight into the nature of the super-heavy isotopes. Applying $Q_{\alpha}$ values as outlined in eq. (7) have a number of advantages. Not only are they measured to a higher nucleon number, but eq. (7) only employs three different measurements, which gives a more compact relation, more likely to be applicable. Unfortunately, the measured $Q_{\alpha}$ values do not necessarily relate to ground state configurations. The specific state, in particular, among the super-heavy isotopes are usually unknown. This fact alone makes it very difficult to extrapolate binding energies accurately from chains of connected $Q_{\alpha}$ measurements. As a consequence we shall only use the results to shed light on the general tendencies of the binding energies in the super-heavy region.

The results for the super-heavy region are displayed in fig. 4 for both $\Delta_{2 \alpha}$ and $\Delta_{2 n}$ relations. We notice first of all a rather clear picture of the deviation from zero around the known high-end shells at $N=126$ and $Z=$ 82. The size of the deviations is $\sim 2-4 \mathrm{MeV}$ both positive and negative.

Otherwise, the most interesting feature in fig. 4, visible with both $\Delta_{2 \alpha}$ and $\Delta_{2 n}$, is the systematic non-zero values around $N=152$. This deviation extend through all evaluations with $\Delta_{2 \alpha}$, and with $\Delta_{2 n}$ significant positive deviations are also visible. This is exactly the behaviour expected from a minor shell, and with $\Delta_{2 n}$ the deviations are even symmetric around the shell. The size of the deviations are $\sim 1 \mathrm{MeV}$, so it is rather weak compared with other shells. This is not surprising considering the nucleon number it occurs at, but these features definitely corresponds to that of an ordinary shell. On the other hand, no other shell effect appears even though the continued increasing stability demonstrate that some shell effects provide the necessary smaller binding energy.

\section{EXTRAPOLATED BINDING ENERGIES}

We shall compare the results from different extrapolations, and define a suitable average leading to better accuracy. Any systematic discrepancies between the individual relations should be accentuated by such a combination. Particularly interesting are applications in the region where $Z>82$ and $N<126$, which is less known. The estimations presented by Audi and Meng 21] for instance diligently cover most of the chart of nuclides except for this specific area. After the general discussion we shall provide tables of extrapolated nuclear binding energies.

\section{A. Improving the accuracy}

The individual extrapolations are all legitimate attempts at estimating the binding energy of unknown isotopes. In the landscape of binding energies each linear mass relation can be seen as approaching the unknown isotope from a different direction. A single mass relation cannot be expected to provide perfect predictions, because of the fluctuating, possibly chaotic, nature of the binding energy. However, if a particular isotope could be approached from several different directions, the expected fluctuations could be viewed from several sides, which would provide a clearer image of the given isotope. In other words, if an isotope could be extrapolated by several different mass relations, the results could be examined and used either to select the most accurate of the extrapolations or combined to provide a much more reliable estimate of the binding energy.

Comparing different extrapolations would also examine the legitimacy of the method itself. If different extrapolations for the same isotope deviated significantly it could cast doubt on the entire procedure. Different mass relations will, of course, involve shells or other influencing factors at different isotopes, and care must be taken when comparing the extrapolations.

There are several considerations to bear in mind, when combining extrapolations based on different mass relations. Both when selecting or prioritizing particular extrapolations, and when calculating an appropriate uncertainty for the final result. To avoid confusion the exact procedure leading to the recommended results will first be explained in some detail.

First of all, it is vital that the individual extrapolations seem reliable, and have a certain degree of precision. To accommodate these requirements only extrapolations with a limited uncertainty, specifically extrapolations where $\sigma_{i}<500 \mathrm{keV}$, are included in the calculated average. However, to demonstrate the possible fallibility of the individual mass relations in certain areas, all extrapolations of relevant isotopes, even if unused, are included in table 【. The final column in table \lists the mass relations used in the calculated average.

The actual weighted average is calculated in stages to both account for known effects such as shells and to detect general deviations. First we use all extrapolations with $\sigma_{i}<500 \mathrm{keV}$ to provide $r_{i} \pm \sigma_{i}$. Second, we define the relation

$$
f_{i}=\frac{\left|r_{i}-r\right|}{\sigma_{i}}
$$

where $r$ denotes the average, and $r_{i}$ is the individual extrapolation. If $\max \left(f_{i}\right)<3 / 2$, then the individual extrapolations are within an acceptable range of the aver- 

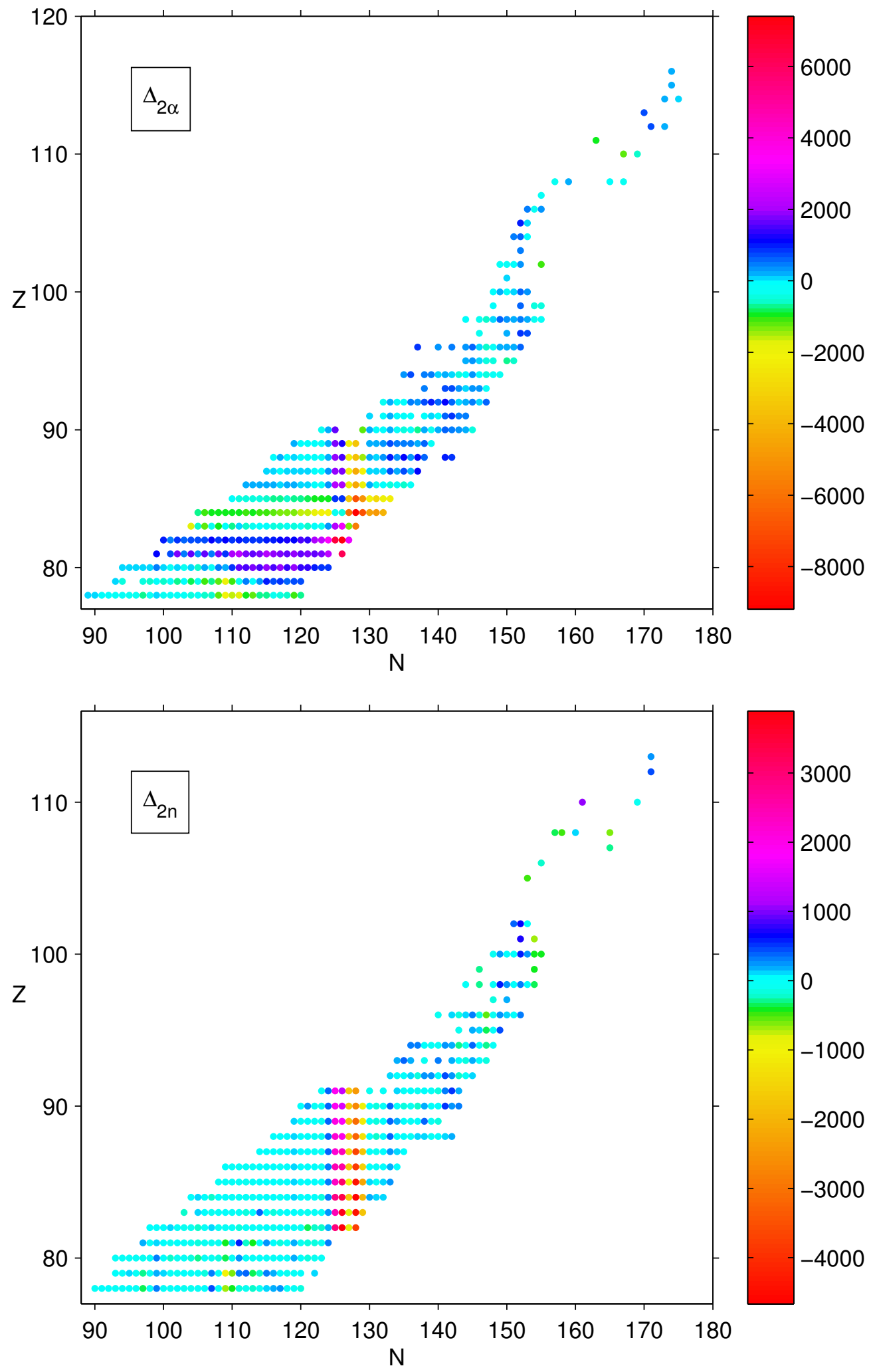

Figure 4. Results of evaluating $Q_{\alpha}$ values with the $\Delta_{2 \alpha}$ relation above and the $\Delta_{2 n}$ relation below. The evaluations are confined to the superheavy isotopes. The colour scale is still in $\mathrm{keV}$. 
age, and the uncertainty for the average value is defined as $\sigma=\min \left(\sigma_{i}\right)$.

Otherwise, if $\max \left(f_{i}\right)>3 / 2$ the individual extrapolations differ too greatly from the average result, and the computed average has little meaning. Any extrapolations involving shell crossings or crossing the $N=Z$ line often differs from the general tendencies, as apparent from figs. 2 and 3 . These results are marked with a * symbol in table If and are considered less reliable. The marked extrapolations are then excluded, and a new, more plausible, average is calculated. Based on this average a relation similar to the one presented in eq. (9) is defined, though now obtained by fewer extrapolations. Once again, if $\max \left(f_{i}\right)<3 / 2$, then the uncertainty is defined as $\sigma=\min \left(\sigma_{i}\right)$.

The extrapolations may be incompatible as in the neighbourhood of closed shells or by crossing the $N=Z$ line. Then a meaningful uncertainty is defined as $\sigma=$ $2 / 3 \max \left(\left|r_{i}-r\right|\right)$, where $r_{i}$ only includes the extrapolations used in the final calculation of $r$. As a consequence of this procedure some results might be based on a single extrapolation, even though multiple mass relations have estimated the isotope. The other available extrapolations could for instance involve a shell crossing, and would then be discarded if the initial results were incompatible. Actually, some isotopes, which have been estimated by several mass relations, might not have a meaningful resulting average at all, if all the extrapolations had an uncertainty exceeding $500 \mathrm{keV}$. The isotopes in both cases, those with either an average based on a single extrapolation or no average at all, have been omitted from table [1] as they provided no relevant information.

\begin{tabular}{|c|c|c|c|c|c|c|c|}
\hline \multirow{2}{*}{$\begin{array}{l}\text { Nucleus } \\
(\mathrm{Z}, \mathrm{N})\end{array}$} & \multicolumn{5}{|c|}{ Estimates of binding energies [keV] } & Average $[\mathrm{keV}]$ & \multirow[t]{2}{*}{ Applied Relations } \\
\hline & $\Delta_{2}$ & & $\Delta_{2 p}$ & $\Delta_{2 \alpha}$ & $\Delta_{2(N-Z)}$ & & \\
\hline$(26,43)$ & 576250 & \pm 1292 & $* 577468 \pm 239$ & $* 577029 \pm 202$ & $* 578091 \pm 275$ & $577422 \pm 446$ & $\Delta_{2 p}, \Delta_{2 \alpha}, \Delta_{2(N-Z)}$ \\
\hline$(26,44)$ & 579206 & \pm 2256 & $* 582840 \pm 250$ & - & $* 584436 \pm 276$ & $583561 \pm 583$ & $\Delta_{2 p}, \Delta_{2(N-Z)}$ \\
\hline$(27,45)$ & 597521 & \pm 2633 & $* 601637 \pm 257$ & $* 601272 \pm 152$ & $* 601465 \pm 361$ & $601378 \pm 152$ & $\Delta_{2 p}, \Delta_{2 \alpha}, \Delta_{2(N-Z)}$ \\
\hline$(28,46)$ & 623965 & \pm 648 & $* 623861 \pm 297$ & $* 619868 \quad \pm 2212$ & $* 624385 \pm 203$ & $624218 \pm 203$ & $\Delta_{2 p}, \Delta_{2(N-Z)}$ \\
\hline$(32,55)$ & *719433 & \pm 237 & $721976 \pm 1353$ & - & $* 718789 \pm 410$ & $719272 \pm 237$ & $\Delta_{2 n}, \Delta_{2(N-Z)}$ \\
\hline$(33,55)$ & *733407 & \pm 256 & $735628 \pm 1530$ & $738781 \pm 2351$ & $* 732688 \pm 394$ & $733193 \pm 256$ & $\Delta_{2 n}, \Delta_{2(N-Z)}$ \\
\hline$(36,33)$ & 561880 & \pm 486 & $561002 \pm 1513$ & - & $565048 \pm 294$ & $564201 \pm 1548$ & $\Delta_{2 n}, \Delta_{2(N-Z)}$ \\
\hline$(36,34)$ & $* 580478$ & \pm 192 & $* 582467 \quad \pm 2134$ & - & $580221 \pm 276$ & $580395 \pm 192$ & $\Delta_{2 n}, \Delta_{2(N-Z)}$ \\
\hline$(36,62)$ & 806662 & \pm 366 & $807701 \pm 185$ & - & $805037 \pm 1328$ & $807490 \pm 552$ & $\Delta_{2 n}, \Delta_{2 p}$ \\
\hline$(36,63)$ & 808943 & \pm 422 & $811736 \pm 540$ & - & $811516 \pm 78$ & $811431 \pm 1659$ & $\Delta_{2 n}, \Delta_{2(N-Z)}$ \\
\hline$(37,35)$ & $* 592557$ & \pm 489 & $* 594706 \quad \pm 1896$ & - & $593650 \pm 362$ & $593264 \pm 362$ & $\Delta_{2 n}, \Delta_{2(N-Z)}$ \\
\hline$(38,64)$ & 846621 & \pm 1136 & $845897 \pm 155$ & - & $846966 \pm 256$ & $846184 \pm 521$ & $\Delta_{2 p}, \Delta_{2(N-Z)}$ \\
\hline$(40,38)$ & - & & $* 642144 \pm 166$ & - & $642129 \pm 151$ & $642136 \pm 151$ & $\Delta_{2 p}, \Delta_{2(N-Z)}$ \\
\hline$(40,42)$ & 694171 & \pm 4774 & $694590 \pm 230$ & $694624 \pm 145$ & $696013 \pm 1606$ & $694614 \pm 145$ & $\Delta_{2 p}, \Delta_{2 \alpha}$ \\
\hline$(42,70)$ & 928505 & \pm 382 & $928726 \quad \pm 2999$ & - & $929351 \pm 271$ & $929068 \pm 271$ & $\Delta_{2 n}, \Delta_{2(N-Z)}$ \\
\hline$(45,74)$ & 988066 & \pm 193 & $* 986373 \pm 1081$ & $* 986234 \quad \pm 1019$ & $* 987801 \pm 216$ & $987949 \pm 193$ & $\Delta_{2 n}, \Delta_{2(N-Z)}$ \\
\hline$(45,75)$ & 991514 & \pm 260 & $* 990420 \pm 1214$ & $* 991092 \quad \pm 2681$ & $* 991504 \pm 176$ & $991507 \pm 176$ & $\Delta_{2 n}, \Delta_{2(N-Z)}$ \\
\hline$(48,48)$ & *797281 & \pm 190 & $* 793119 \pm 175$ & - & $* 795277 \quad \pm 1121$ & $795033 \pm 1499$ & $\Delta_{2 n}, \Delta_{2 p}$ \\
\hline$(48,83)$ & - & & $* 1080376 \pm 381$ & - & $* 1084581 \pm 197$ & $1083690 \pm 2209$ & $\Delta_{2 p}, \Delta_{2(N-Z)}$ \\
\hline$(49,84)$ & *1097714 & \pm 232 & $* 1095129 \pm 400$ & - & $* 1102390 \pm 87$ & $1101543 \pm 4276$ & $\Delta_{2 n}, \Delta_{2 p}, \Delta_{2(}$ \\
\hline$(50,51)$ & *835352 & \pm 287 & $* 836267 \pm 295$ & - & $* 835137 \pm 117$ & $835299 \pm 646$ & $\Delta_{2 n}, \Delta_{2 p}, \Delta_{2(N-Z)}$ \\
\hline$(52,52)$ & $* 848264$ & \pm 323 & $* 852185 \pm 353$ & - & $* 850904 \pm 194$ & $850562 \pm 1532$ & $\Delta_{2 n}, \Delta_{2 p}, \Delta_{2(N-Z)}$ \\
\hline$(52,87)$ & $* 1138134$ & \pm 1243 & $1140623 \pm 270$ & - & $* 1140110 \pm 265$ & $1140362 \pm 265$ & $\Delta_{2 p}, \Delta_{2(N-Z)}$ \\
\hline$(53,85)$ & $* 1144407$ & \pm 1509 & $1143992 \pm 221$ & $1144890 \pm 266$ & $* 1142366 \quad \pm 2237$ & $1144358 \pm 355$ & $\Delta_{2 p}, \Delta_{2 \alpha}$ \\
\hline$(58,94)$ & 1240393 & \pm 202 & $1241112 \pm 409$ & - & $1242205 \pm 155$ & $1241501 \pm 738$ & $\Delta_{2 n}, \Delta_{2 p}, \Delta_{2}$ \\
\hline$(60,68)$ & 1047404 & \pm 187 & $1049530 \pm 2129$ & - & $1046496 \pm 93$ & $1046674 \pm 487$ & $\Delta_{2 n}, \Delta_{2(N-Z)}$ \\
\hline$(60,95)$ & 1268120 & \pm 682 & $1266702 \pm 277$ & $1266251 \pm 423$ & $1266860 \pm 771$ & $1266566 \pm 277$ & $\Delta_{2 p}, \Delta_{2 \alpha}$ \\
\hline$(61,70)$ & 1070175 & \pm 395 & $1070384 \pm 762$ & - & $1068999 \pm 333$ & $1069488 \pm 458$ & $\Delta_{2 n}, \Delta_{2(N-Z)}$ \\
\hline$(61,71)$ & 1079011 & \pm 1534 & $1080538 \pm 1146$ & $1079524 \pm 388$ & $1079127 \pm 303$ & $1079277 \pm 303$ & $\Delta_{2 \alpha}, \Delta_{2(N-Z)}$ \\
\hline$(62,70)$ & & & $1072374 \pm 446$ & - & $1072246 \pm 122$ & $1072254 \pm 122$ & $\Delta_{2 p}, \Delta_{2(N-Z)}$ \\
\hline$(62,71)$ & 1081835 & \pm 1800 & $1081867 \pm 393$ & - & $1082341 \pm 181$ & $1082257 \pm 181$ & $\Delta_{2 p}, \Delta_{2(N-Z)}$ \\
\hline$(62,72)$ & 1094145 & \pm 2024 & $1094109 \pm 362$ & $1094870 \pm 209$ & $1094296 \pm 165$ & $1094471 \pm 266$ & $\Delta_{2 p}, \Delta_{2 \alpha}, \Delta_{2(N}$ \\
\hline$(62,98)$ & 1302650 & \pm 448 & $1302344 \pm 214$ & - & $1302713 \pm 1063$ & $1302401 \pm 214$ & $\Delta_{2 n}, \Delta_{2 p}$ \\
\hline$(63,72)$ & - & & $1094922 \pm 283$ & - & $1093656 \pm 499$ & $1094614 \pm 638$ & $\Delta_{2 p}, \Delta_{2(N-Z)}$ \\
\hline$(63,74)$ & 1117498 & \pm 3591 & $1116361 \pm 210$ & $1117409 \pm 344$ & $1115580 \pm 695$ & $1116645 \pm 509$ & $\Delta_{2 p}, \Delta_{2 \alpha}$ \\
\hline$(64,74)$ & 1120095 & \pm 3568 & $1119918 \pm 109$ & - & $1119650 \pm 144$ & $1119820 \pm 109$ & $\Delta_{2 p}, \quad \Delta_{2(N-Z)}$ \\
\hline$(64,100)$ & 1333063 & \pm 248 & $1332671 \pm 328$ & $1333134 \pm 489$ & $1334078 \pm 641$ & $1332950 \pm 248$ & $\Delta_{2 n}, \Delta_{2 p}, \Delta_{2 \alpha}$ \\
\hline$(64,101)$ & 1337977 & \pm 453 & $1337702 \pm 295$ & - & $1338532 \pm 730$ & $1337784 \pm 295$ & $\Delta_{2 n}, \Delta_{2 p}$ \\
\hline$(65,100)$ & 1341837 & \pm 190 & $1341306 \pm 491$ & $1339899 \pm 757$ & $1342848 \pm 424$ & $1341928 \pm 613$ & $\Delta_{2 n}, \Delta_{2 p}, \Delta_{2(N-Z)}$ \\
\hline$(67,105)$ & 1387302 & \pm 333 & $1387183 \pm 498$ & $1385586 \pm 1250$ & $1388720 \pm 385$ & $1387762 \pm 639$ & $\Delta_{2 n}, \Delta_{2 p}, \Delta_{2(N-Z)}$ \\
\hline$(68,105)$ & 1396784 & \pm 436 & $1398240 \pm 323$ & $1397201 \pm 58$ & $1398343 \pm 161$ & $1397349 \pm 663$ & $\Delta_{2 n}, \Delta_{2 p}, \Delta_{2 \alpha}, \Delta_{2(N-Z)}$ \\
\hline$(68,106)$ & 1403282 & \pm 249 & $1404464 \pm 609$ & $1404991 \pm 357$ & $1404826 \pm 129$ & $1404544 \pm 841$ & $\Delta_{2 n}, \Delta_{2 \alpha}, \Delta_{2(N-Z)}$ \\
\hline
\end{tabular}




\begin{tabular}{|c|c|c|c|c|c|c|}
\hline \multirow{2}{*}{$\begin{array}{l}\text { Nucleus } \\
(\mathrm{Z}, \mathrm{N})\end{array}$} & \multicolumn{4}{|c|}{ Estimates of binding energies [keV] } & \multirow[t]{2}{*}{ Average $[\mathrm{keV}]$} & \multirow[t]{2}{*}{ Applied Relations } \\
\hline & $\Delta_{2 n}$ & $\Delta_{2 p}$ & $\Delta_{2 \alpha}$ & $\Delta_{2(N-Z)}$ & & \\
\hline$(69,108)$ & $1422022 \pm 184$ & $1423137 \pm 998$ & - & $1422893 \pm 93$ & $1422716 \pm 463$ & $\Delta_{2 n}, \Delta_{2(N-Z)}$ \\
\hline$(69,109)$ & $1426615 \pm 361$ & $1428561 \pm 1044$ & - & $1427412 \pm 474$ & $1426908 \pm 361$ & $\Delta_{2 n}, \Delta_{2(N-Z)}$ \\
\hline$(70,109)$ & $1436405 \pm 360$ & $1435954 \pm 1439$ & $1436501 \pm 103$ & $1435908 \pm 635$ & $1436494 \pm 103$ & $\Delta_{2 n}, \Delta_{2 \alpha}$ \\
\hline$(71,83)$ & $* 1227402 \pm 438$ & $1227271 \pm 471$ & - & $* 1226192 \pm 779$ & $1227341 \pm 438$ & $\Delta_{2 n}, \Delta_{2 p}$ \\
\hline$(71,110)$ & $1450595 \pm 425$ & $1448160 \pm 1502$ & $1450074 \pm 93$ & $1450005 \pm 593$ & $1450097 \pm 93$ & $\Delta_{2 n}, \Delta_{2 \alpha}$ \\
\hline$(72,85)$ & $1249894 \pm 187$ & $1249664 \pm 319$ & - & $1250965 \pm 638$ & $1249835 \pm 187$ & $\Delta_{2 n}, \Delta_{2 p}$ \\
\hline$(73,85)$ & $1250003 \pm 404$ & $1249843 \pm 497$ & - & $1250054 \pm 235$ & $1250013 \pm 235$ & $\Delta_{2 n}, \Delta_{2 p}, \Delta_{2(N-Z)}$ \\
\hline$(74,87)$ & $1271494 \pm 365$ & $1272211 \pm 113$ & - & $1272571 \pm 361$ & $1272183 \pm 459$ & $\Delta_{2 n}, \Delta_{2 p}, \Delta_{2(N-Z)}$ \\
\hline$(75,87)$ & $1271867 \pm 492$ & $1271718 \pm 302$ & - & $1270835 \pm 507$ & $1271759 \pm 302$ & $\Delta_{2 n}, \Delta_{2 p}$ \\
\hline$(75,92)$ & $1324297 \pm 207$ & $1324669 \pm 311$ & $1323990 \pm 403$ & $1325523 \pm 1207$ & $1324346 \pm 207$ & $\Delta_{2 n}, \Delta_{2 p}, \Delta_{2 \alpha}$ \\
\hline$(75,118)$ & $1529546 \pm 345$ & $* 1529841 \quad \pm 1167$ & $* 1530772 \quad \pm 2269$ & $* 1530133 \pm 429$ & $1529776 \pm 345$ & $\Delta_{2 n}, \Delta_{2(N-Z)}$ \\
\hline$(76,89)$ & $1293730 \pm 390$ & $1293974 \pm 229$ & - & $1294570 \pm 340$ & $1294078 \pm 229$ & $\Delta_{2 n}, \Delta_{2 p}, \Delta_{2(N-Z)}$ \\
\hline$(77,88)$ & $1282791 \pm 307$ & $1283328 \pm 429$ & - & $1281841 \pm 531$ & $1282973 \pm 307$ & $\Delta_{2 n}, \Delta_{2 p}$ \\
\hline$(77,93)$ & $1335380 \pm 180$ & $1335618 \pm 220$ & $1335199 \pm 400$ & $1336152 \pm 643$ & $1335446 \pm 180$ & $\Delta_{2 n}, \Delta_{2 p}, \Delta_{2 \alpha}$ \\
\hline$(78,91)$ & $1314656 \pm 761$ & $1315246 \pm 242$ & - & $1314896 \pm 292$ & $1315104 \pm 242$ & $\Delta_{2 p}, \Delta_{2(N-Z)}$ \\
\hline$(79,90)$ & $1303813 \pm 240$ & $1304075 \pm 282$ & - & $1303200 \pm 380$ & $1303787 \pm 391$ & $\Delta_{2 n}, \Delta_{2 p}, \Delta_{2(N-Z)}$ \\
\hline$(79,95)$ & $1356317 \pm 297$ & $1356279 \pm 181$ & - & $1355432 \pm 528$ & $1356290 \pm 181$ & $\Delta_{2 n}, \Delta_{2 p}$ \\
\hline$(80,93)$ & $1335989 \pm 479$ & $1336045 \pm 258$ & - & $1336382 \pm 448$ & $1336104 \pm 258$ & $\Delta_{2 n}, \Delta_{2 p}, \Delta_{2(N-Z)}$ \\
\hline$(80,131)$ & - & $* 1644785 \pm 366$ & - & $* 1643683 \pm 294$ & $1644115 \pm 447$ & $\Delta_{2 p}, \Delta_{2(N-Z)}$ \\
\hline$(81,97)$ & $1377933 \pm 406$ & $* 1377553 \pm 446$ & - & $* 1377229 \pm 220$ & $1377415 \pm 220$ & $\Delta_{2 n}, \Delta_{2 p}, \Delta_{2(N-Z)}$ \\
\hline$(82,94)$ & $1346745 \pm 126$ & $* 1347085 \pm 243$ & $* 1346991 \quad \pm 1303$ & $* 1347653 \pm 636$ & $1346817 \pm 126$ & $\Delta_{2 n}, \Delta_{2 p}$ \\
\hline$(82,95)$ & $1356385 \pm 434$ & $* 1356892 \pm 452$ & - & $* 1357465 \pm 853$ & $1356628 \pm 434$ & $\Delta_{2 n}, \Delta_{2 p}$ \\
\hline$(83,98)$ & - & $* 1389816 \pm 321$ & $* 1390047 \pm 488$ & $* 1391306 \quad \pm 1084$ & $1389886 \pm 321$ & $\Delta_{2 p}, \Delta_{2 \alpha}$ \\
\hline$(84,96)$ & - & $* 1366448 \pm 262$ & - & $* 1366742 \pm 156$ & $1366665 \pm 156$ & $\Delta_{2 p}, \Delta_{2(N-Z)}$ \\
\hline$(84,98)$ & - & $* 1389136 \pm 311$ & $* 1388890 \pm 132$ & $* 1389155 \pm 80$ & $1389087 \pm 80$ & $\Delta_{2 p}, \Delta_{2 \alpha}, \Delta_{2(N-Z)}$ \\
\hline$(84,99)$ & - & $* 1399474 \pm 424$ & *1398674 \pm 463 & $* 1398201 \pm 227$ & $1398514 \pm 640$ & $\Delta_{2 p}, \Delta_{2 \alpha}, \Delta_{2(N-Z)}$ \\
\hline$(84,140)$ & - & $1712129 \pm 227$ & - & $1711176 \pm 253$ & $1711704 \pm 352$ & $\Delta_{2 p}, \Delta_{2(N-Z)}$ \\
\hline$(84,141)$ & - & $1715816 \pm 384$ & - & $1714216 \pm 141$ & $1714407 \pm 939$ & $\Delta_{2 p}, \Delta_{2(N-Z)}$ \\
\hline$(84,142)$ & - & $1720838 \pm 290$ & - & $1719998 \pm 55$ & $1720027 \pm 541$ & $\Delta_{2 p}, \Delta_{2(N-Z)}$ \\
\hline$(84,143)$ & - & $1724348 \pm 237$ & - & $1723840 \pm 270$ & $1724127 \pm 237$ & $\Delta_{2 p}, \Delta_{2(N-Z)}$ \\
\hline$(86,103)$ & - & $* 1438847 \pm 349$ & - & $* 1438645 \quad \pm 271$ & $1438721 \pm 271$ & $\Delta_{2 p}, \Delta_{2(N-Z)}$ \\
\hline$(86,144)$ & $1747230 \pm 93$ & $1747198 \pm 173$ & - & $1746714 \pm 170$ & $1747127 \pm 276$ & $\Delta_{2 n}, \Delta_{2 p}, \Delta_{2(N-Z)}$ \\
\hline$(86,145)$ & $1751216 \pm 89$ & - & - & $1750244 \pm 110$ & $1750834 \pm 393$ & $\Delta_{2 n}, \Delta_{2(N-Z)}$ \\
\hline$(87,111)$ & $1520866 \pm 442$ & $* 1519842 \quad \pm 1133$ & $* 1520530 \pm 312$ & $* 1520839 \quad \pm 1472$ & $1520642 \pm 312$ & $\Delta_{2 n}, \Delta_{2 \alpha}$ \\
\hline$(88,146)$ & $1772938 \pm 147$ & - & - & $1771596 \pm 496$ & $1772829 \pm 822$ & $\Delta_{2 n}, \Delta_{2(N-Z)}$ \\
\hline$(90,116)$ & $1572224 \pm 841$ & $1572457 \pm 1181$ & $1572136 \pm 212$ & $1572279 \pm 167$ & $1572224 \pm 167$ & $\Delta_{2 \alpha}, \Delta_{2(N-Z)}$ \\
\hline$(90,117)$ & $1580086 \pm 1803$ & $1581275 \pm 1135$ & $1580703 \pm 450$ & $1580524 \pm 489$ & $1580621 \pm 450$ & $\Delta_{2 \alpha}, \Delta_{2(N-Z)}$ \\
\hline$(90,147)$ & $1791915 \pm 232$ & - & - & $1792436 \pm 256$ & $1792150 \pm 232$ & $\Delta_{2 n}, \Delta_{2(N-Z)}$ \\
\hline$(92,123)$ & - & $1641179 \pm 2031$ & $1638388 \pm 342$ & *1639006 \pm 358 & $1638683 \pm 342$ & $\Delta_{2 \alpha}, \Delta_{2(N-Z)}$ \\
\hline$(92,149)$ & $1816700 \pm 422$ & - & $1816384 \pm 247$ & - & $1816465 \pm 247$ & $\Delta_{2 n}, \Delta_{2 \alpha}$ \\
\hline$(94,131)$ & - & $1706355 \pm 424$ & - & $1705431 \pm 131$ & $1705512 \pm 562$ & $\Delta_{2 p}, \Delta_{2(N-Z)}$ \\
\hline$(94,132)$ & $1714752 \pm 162$ & $1714640 \pm 303$ & - & $1714508 \pm 50$ & $1714531 \pm 50$ & $\Delta_{2 n}, \Delta_{2 p}, \Delta_{2(}$ \\
\hline$(94,154)$ & $1856744 \pm 85$ & $1856600 \pm 359$ & - & - & $1856736 \pm 85$ & $\Delta_{2 n}, \Delta_{2 p}$ \\
\hline$(95,132)$ & - & $1715359 \pm 480$ & - & $1714860 \pm 254$ & $1714969 \pm 254$ & $\Delta_{2 p}, \Delta_{2(N-Z)}$ \\
\hline$(96,134)$ & - & $1734758 \pm 458$ & - & $1734312 \pm 174$ & $1734368 \pm 174$ & $\Delta_{2 p}, \Delta_{2(N-Z)}$ \\
\hline$(96,136)$ & $1751675 \pm 294$ & - & $1752023 \pm 382$ & $1750297 \pm 415$ & $1751444 \pm 765$ & $\Delta_{2 n}, \Delta_{2 \alpha}, \Delta_{2(N-Z)}$ \\
\hline$(98,140)$ & $1786792 \pm 243$ & $1787021 \pm 237$ & $1786900 \pm 712$ & - & $1786909 \pm 237$ & $\Delta_{2 n}, \Delta_{2 p}$ \\
\hline$(100,144)$ & $1822301 \pm 819$ & $1821948 \pm 172$ & $1822088 \pm 327$ & - & $1821979 \pm 172$ & $\Delta_{2 p}, \Delta_{2 \alpha}$ \\
\hline
\end{tabular}

Table I: The results of combining the extrapolations based on the four different mass relations. The final column indicates which relations were used when calculating a particular average. Only extrapolations with an uncertainty $\sigma_{i}<500 \mathrm{keV}$ were considered when calculating this average. If an extrapolation includes isotopes influence by either a shell or on the $N=Z$ line, it is marked with a symbol.

\section{B. Numerical results}

All the results presented in table 1 have merit and provide some information, though not all will be commented on. Instead, focus will be on a select few, which demon- strates the various considerations necessary when evaluating the results. Though, some general propensities can be seen by observing the results as a whole.

It is immediately obvious that extrapolations based on $\Delta_{2 \alpha}$ feature a lot less frequently than any of the other mass relations. The reason is that $\Delta_{2 \alpha}$ extrapolates along 
the stability curve and towards the super-heavy nuclei. This makes it difficult to compare $\Delta_{2 \alpha}$ with any of the other relations as they rarely coincide. From table $\mathrm{I}$ it is also evident that most results are calculated based on only two different mass relations. Of course, a combination based on additional extrapolations would be preferable, but even if just two are comparable the result's credibility would increase greatly.

The applicability of the mass relations in various areas across the chart of nuclides is demonstrated by the scattered results. Viewing this erratic distribution col- lectively, it is clear that the results are more consistent with a greater degree of certainty among the heavier isotopes. The difference between extrapolations with lighter isotopes are typically $\sim 800 \mathrm{keV}$, whereas the heavier isotopes often differ with less than $400 \mathrm{keV}$. There are also extrapolations with very large differences, but these are almost exclusively found among the light nuclei. This tendency was to be expected as the binding energy per nucleon generally varies more for lighter isotopes.

\begin{tabular}{|c|c|c|c|c|c|c|c|c|}
\hline \multirow{2}{*}{$\begin{array}{l}\text { Nucleus } \\
(\mathrm{Z}, \mathrm{N})\end{array}$} & \multicolumn{5}{|c|}{ Estimates of binding energies $[\mathrm{keV}]$} & \multirow{2}{*}{$\begin{array}{c}\text { Measured } \\
{[\mathrm{keV}]}\end{array}$} & \multirow{2}{*}{$\frac{\text { Difference }}{\mathrm{A} \& \mathrm{M}}$} & \multirow{2}{*}{$\frac{s[\mathrm{keV}]}{\text { Exp. }}$} \\
\hline & \multicolumn{2}{|c|}{ Average } & \multicolumn{3}{|c|}{ Audi and Meng } & & & \\
\hline$(26,43)$ & 577422 & $\pm \quad 446$ & 574977 & \pm & 483 & - & 2445 & - \\
\hline$(26,44)$ & 583561 & 583 & 580930 & \pm & 630 & - & 2631 & - \\
\hline$(27,45)$ & 601378 & 152 & 599688 & \pm & 576 & - & 1690 & - \\
\hline$(28,46)$ & 624218 & 203 & 624042 & \pm & 370 & - & 176 & - \\
\hline$(32,55)$ & 719272 & 237 & 721404 & \pm & 522 & - & -2132 & - \\
\hline$(33,55)$ & 733193 & $\pm \quad 256$ & 735328 & \pm & 440 & - & -2135 & . \\
\hline$(36,33)$ & 564201 & \pm 1548 & 561177 & \pm & 414 & - & 3024 & - \\
\hline$(36,34)$ & 580395 & $\pm \quad 192$ & 578410 & \pm & 350 & - & 1985 & - \\
\hline$(36,62)$ & 807490 & $\pm \quad 552$ & 807324 & \pm & 490 & - & 166 & - \\
\hline$(36,63)$ & 811431 & \pm 1659 & 809622 & \pm & 495 & - & 1809 & 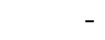 \\
\hline$(37,35)$ & 593264 & $\pm \quad 362$ & 590328 & \pm & 504 & - & 2936 & - \\
\hline$(38,64)$ & 846184 & $\pm \quad 521$ & 845988 & \pm & 204 & $845904 \pm 70$ & 196 & 280 \\
\hline$(40,38)$ & 642136 & 151 & 639600 & \pm & 468 & - & 2536 & - \\
\hline$(40,42)$ & 694614 & 145 & 694458 & \pm & 164 & - & 156 & - \\
\hline$(42,70)$ & 929068 & 271 & 928704 & \pm & 336 & - & 364 & 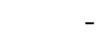 \\
\hline$(45,74)$ & 987949 & 193 & 988176 & \pm & 238 & $988104 \pm 9$ & -227 & -155 \\
\hline$(45,75)$ & 991507 & 176 & 992160 & \pm & 240 & - & -653 & - \\
\hline$(48,48)$ & 795033 & \pm 1499 & 792864 & \pm & 384 & - & 2169 & - \\
\hline$(48,83)$ & 1083690 & \pm 2209 & 1075117 & \pm & 131 & - & 8573 & - \\
\hline$(49,84)$ & 1101543 & \pm 4276 & 1092861 & \pm & 266 & - & 8682 & - \\
\hline$(50,51)$ & 835299 & $\pm \quad 646$ & 835977 & \pm & 303 & $836391 \pm 300$ & -678 & -1092 \\
\hline$(52,87)$ & 1140362 & $\pm \quad 265$ & 1141607 & \pm & 417 & $1141436 \pm 4$ & -1245 & -1074 \\
\hline$(53,85)$ & 1144358 & 355 & 1144296 & \pm & 138 & $1144357 \pm 6$ & 62 & 1 \\
\hline$(58,94)$ & 1241501 & 738 & 1240776 & \pm & 152 & - & 725 & - \\
\hline$(60,68)$ & 1046674 & 487 & 1046272 & \pm & 256 & - & 402 & _ \\
\hline$(60,95)$ & 1266566 & 277 & 1266505 & \pm & 155 & $1266398 \pm 16$ & 61 & 168 \\
\hline$(61,70)$ & 1069488 & 458 & 1069222 & \pm & 131 & - & 266 & - \\
\hline$(61,71)$ & 1079277 & 303 & 1079364 & \pm & 132 & - & -87 & - \\
\hline$(62,70)$ & 1072254 & 122 & 1071576 & \pm & 264 & - & 678 & - \\
\hline$(62,71)$ & 1082257 & 181 & 1081822 & \pm & 133 & - & 435 & . \\
\hline$(62,72)$ & 1094471 & 266 & 1094244 & \pm & 134 & - & 227 & - \\
\hline$(62,98)$ & 1302401 & 214 & 1303360 & \pm & 160 & $1303142 \pm 10$ & -959 & -741 \\
\hline$(63,72)$ & 1094614 & \pm 638 & 1094445 & \pm & 270 & - & 169 & - \\
\hline$(63,74)$ & 1116645 & 509 & 1116550 & \pm & 137 & - & 95 & - \\
\hline$(64,74)$ & 1119820 & 109 & 1119456 & \pm & 138 & - & 364 & - \\
\hline$(64,100)$ & 1332950 & 248 & 1333484 & \pm & 328 & - & -534 & 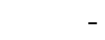 \\
\hline$(64,101)$ & 1337784 & \pm 295 & 1338315 & \pm & 495 & - & -531 & - \\
\hline$(65,100)$ & 1341928 & 613 & 1341615 & \pm & 165 & - & 313 & - \\
\hline$(67,105)$ & 1387762 & $\pm \quad 639$ & 1387352 & \pm & 172 & - & 410 & . \\
\hline$(68,105)$ & 1397349 & $\pm \quad 663$ & 1396802 & \pm & 173 & - & 547 & . \\
\hline$(68,106)$ & 1404544 & \pm 841 & 1403136 & \pm & 348 & - & 1408 & 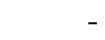 \\
\hline$(69,108)$ & 1422716 & $\pm \quad 463$ & 1422195 & \pm & 354 & - & 521 & - \\
\hline$(69,109)$ & 1426908 & $\pm \quad 361$ & 1426848 & \pm & 356 & - & 60 & - \\
\hline$(70,109)$ & 1436494 & \pm 103 & 1436475 & \pm & 358 & - & 19 & - \\
\hline$(71,83)$ & 1227341 & \pm 438 & 1227072 & \pm & 154 & - & 269 & - \\
\hline$(71,110)$ & 1450097 & \pm & 1450172 & \pm & 362 & $1450159 \pm 159$ & -75 & -62 \\
\hline$(72,85)$ & 1249835 & 187 & 1249563 & \pm & 157 & - & 272 & - \\
\hline$(73,85)$ & 1250013 & $\pm \quad 235$ & 1249148 & \pm & 158 & - & 865 & - \\
\hline
\end{tabular}




\begin{tabular}{|c|c|c|c|c|c|c|c|c|c|}
\hline \multirow{2}{*}{$\begin{array}{l}\text { Nucleus } \\
(\mathrm{Z}, \mathrm{N})\end{array}$} & \multicolumn{6}{|c|}{ Estimates of binding energies $[\mathrm{keV}]$} & \multirow{2}{*}{$\begin{array}{c}\text { Measured } \\
{[\mathrm{keV}]}\end{array}$} & \multirow{2}{*}{$\begin{array}{l}\text { Difference } \\
\text { A \& M }\end{array}$} & \multirow{2}{*}{$\frac{[\mathrm{keV}]}{\text { Exp. }}$} \\
\hline & \multicolumn{3}{|c|}{ Average } & \multicolumn{3}{|c|}{ Audi and Meng } & & & \\
\hline$(74,87)$ & 1272183 & \pm & 459 & 1272061 & \pm & 161 & - & 122 & - \\
\hline$(75,87)$ & 1271759 & \pm & 302 & 1271214 & \pm & 162 & - & 545 & - \\
\hline$(75,92)$ & 1324346 & \pm & 207 & 1324143 & \pm & 0 & - & 203 & - \\
\hline$(75,118)$ & 1529776 & \pm & 345 & 1529332 & \pm & 193 & $1529324 \pm 38$ & 444 & 452 \\
\hline$(76,89)$ & 1294078 & \pm & 229 & 1293930 & \pm & 165 & - & 148 & - \\
\hline$(77,88)$ & 1282973 & \pm & 307 & 1283205 & \pm & 165 & - & -232 & - \\
\hline$(77,93)$ & 1335446 & \pm & 180 & 1335180 & \pm & 170 & - & 266 & - \\
\hline$(78,91)$ & 1315104 & \pm & 242 & 1315327 & \pm & 169 & - & -223 & - \\
\hline$(79,90)$ & 1303787 & \pm & 391 & 1304004 & \pm & 338 & - & -217 & - \\
\hline$(79,95)$ & 1356290 & \pm & 181 & 1356852 & \pm & 174 & - & -562 & - \\
\hline$(80,93)$ & 1336104 & \pm & 258 & 1336252 & \pm & 173 & - & -148 & - \\
\hline$(80,131)$ & 1644115 & \pm & 447 & 1640947 & \pm & 211 & - & 3168 & - \\
\hline$(81,97)$ & 1377415 & \pm & 220 & 1378076 & \pm & 178 & - & -661 & - \\
\hline$(84,140)$ & 1711704 & \pm & 352 & 1712480 & \pm & 224 & - & -776 & - \\
\hline$(84,141)$ & 1714407 & \pm & 939 & 1716075 & \pm & 225 & - & -1668 & - \\
\hline$(84,142)$ & 1720027 & \pm & 541 & 1721216 & \pm & 452 & - & -1189 & - \\
\hline$(84,143)$ & 1724127 & \pm & 237 & 1724519 & \pm & 454 & - & -392 & - \\
\hline$(86,144)$ & 1747127 & \pm & 276 & 1747080 & \pm & 230 & - & 47 & - \\
\hline$(86,145)$ & 1750834 & \pm & 393 & 1750749 & \pm & 231 & - & 85 & - \\
\hline$(88,146)$ & 1772829 & \pm & 822 & 1772784 & \pm & 468 & $1772949 \pm 31$ & 45 & -120 \\
\hline$(90,147)$ & 1792150 & \pm & 232 & 1792194 & \pm & 474 & - & -44 & 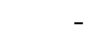 \\
\hline$(92,149)$ & 1816465 & \pm & 247 & 1816899 & \pm & 241 & - & -434 & - \\
\hline$(98,140)$ & 1786909 & \pm & 237 & 1787142 & \pm & 476 & - & -233 & - \\
\hline$(100,144)$ & 1821979 & \pm & 172 & 1822192 & \pm & 244 & - & -213 & - \\
\hline
\end{tabular}

Table II: The average of the extrapolated values from table I compared to estimates by Audi and Meng 21 and to experimental measurements of ten nuclei. The differences between the average and both the estimates and the measurements are listed in the final two columns.

The uncertainty connected to the final average value mostly comes from only one of the relevant extrapolations. This indicates that the extrapolations generally are compatible, and makes the final results more credible. However, some results are questionable, where the uncertainty has been calculated based on the distance between extrapolations. For instance is $\sigma>1 \mathrm{MeV}$ for $(48,48)$, $(48,83),(49,84)$, and $(52,52)$ which makes these results less useful. Such uncertainties are not surprising in view of the involved isotopes, where for example rapidly varying shell effects are pronounced. On the other hand, the significant uncertainty of for instance $(84,141)$ is somewhat more troubling. Considering the involved extrapolations a better result could have been expected. This goes to show the volatility the method in the vicinity of shells, and emphasizes the care that must be taken when analysing these results.

Inconsistent results like those are clearly in the minority, as most have an acceptable uncertainty based on very compatible extrapolations. For instance the averages for $(84,98),(86,103),(86,144),(90,116),(94,132)$ and $(94,154)$ are all based on very consistent extrapolations. The benefit of combining different mass relations is also emphasized when considering $(90,116)$, where $\Delta_{2 n}$ and $\Delta_{2 p}$ have extrapolations with significant uncertainties. The average is then based on $\Delta_{2 \alpha}$ and $\Delta_{2(N-Z)}$, but the final average value is actually consistent with the extrapolations based on $\Delta_{2 n}$ and $\Delta_{2 p}$.

Results where $Z>82$ and $N<126$ are perhaps more interesting. The area defined by these shells has traditionally been difficult to estimate, and isotopes in this area extrapolated by multiple mass relation deserves special attention. Some of these extrapolations involve shells, and must be viewed with suspicion. The nine extrapolations in this area are generally internally consistent, including even those influenced by shells. They must be used with care, but the remaining majority seem to be especially reliable. In particular, the results for $(90,116)$ and $(90,117)$ are based on very close-lying extrapolations, and $(90,116)$ also have a very reasonable uncertainty.

Actually, it could be argued that the procedure is too exclusive in certain situations. For instance the heavier isotopes like $(90,117)$, or in particular $(98,140)$, and $(100,144)$, could possibly have included additional mass relations in the calculations. Here some extrapolations have been excluded based on their uncertainties, even though the final result agrees almost perfectly with these extrapolations. The preferred attitude has been to err on the side of caution, which is why these extrapolations have been excluded.

To get an indication of whether the averages are reasonable extrapolations, they are in table II compared with estimates provided by Audi and Meng [21]. For $Z<64$ the deviation between the estimates are often greater than $1 \mathrm{MeV}$, which again indicates that extrapolations are less reliable for light isotopes. However, for $Z>64$ the differences are usually less than $300 \mathrm{keV}$, and 


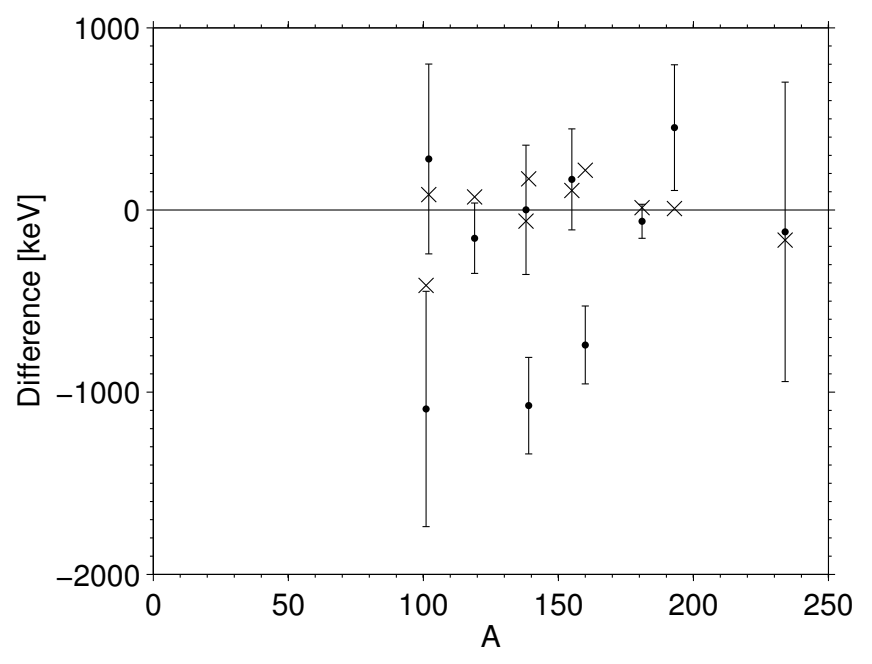

Figure 5. The differences between our extrapolations and the measured values where the errorbars are based solely on the extrapolated uncertainties. The crosses indicate the difference between Audi and Meng's extrapolations and the measurements.

the uncertainties are also very much comparable.

Table [II also includes a comparison with ten nuclei not included in Audi and Meng's preliminary mass table. The measurement of ${ }^{139} \mathrm{Te}$ was done by Hakala et al [22, whereas ${ }^{138} \mathrm{I},{ }^{155} \mathrm{Nd}$ and ${ }^{160} \mathrm{Sm}$ was measured by Van Schelt et al 23. The last six isotopes ${ }^{101} \mathrm{Sn},{ }^{102} \mathrm{Sr}$, ${ }^{119} \mathrm{Rh},{ }^{181} \mathrm{Lu},{ }^{193} \mathrm{Re}$, and ${ }^{234} \mathrm{Ra}$ are included in the final version of Ame2012 [24]. There is a significant difference between the extrapolations of ${ }^{101} \mathrm{Sn},{ }^{139} \mathrm{Te}$ and ${ }^{160} \mathrm{Sm}$ and the measured values. The extrapolations in these cases includes either the shell $Z=50$ or the subshell $Z=64$, which could explain the large deviation. Shell effects could also explain the not insignificant deviation of ${ }^{193}$ Re. On the other hand, the extrapolations of ${ }^{102} \mathrm{Sr}$, ${ }^{119} \mathrm{Rh},{ }^{138} \mathrm{I},{ }^{155} \mathrm{Nd},{ }^{181} \mathrm{Lu}$, and ${ }^{234} \mathrm{Ra}$ are very much comparable to the measured values. Despite the fact that 138 -I are very close to both the $Z=50$ and the $N=82$ shell, the extrapolation predicted exactly the value measured. Apparently, the method can at times be applied near magic numbers.

Fig. 5 provides an overview of the differences between the extrapolated and the measured values as a function of nucleon number. The three nuclei deviating by more than $500 \mathrm{keV}$ in our extrapolation are influenced by closed shell or subshell effects which add to the inaccuracy in the present type of extrapolation. The other more believable points deviate on average by about $200 \mathrm{keV}$.

The corresponding extrapolations by Audi and Meng exhibit a comparable deviation, on average about $130 \mathrm{keV}$. As seen from table III their uncertainties are also comparable. However, shell effects seem included in their extrapolations.

\section{CONCLUSION}

The ultimate purpose of this paper was to extrapolate new binding energies, using several mass relations constructed specifically to this task.

A very general model for describing the binding energy was assumed based on known contributions. Four relations were then designed to eliminate as many factors as possible in the description of the binding energy. The intent was to identify groupings of isotopes where the mass relation either cancelled completely or showed clear, predictable tendencies. By continuing these tendencies outcomes could be predicted, and the binding energy of unknown isotopes could be extrapolated accordingly.

Four mass relations were defined and applied individually. The results were used to confirm the predictions of cancellation of smoothly vanishing aspects, and by extension to corroborate the initial assumption of dividing the binding energy in qualitatively different terms. Each of the mass relations supplied numerous extrapolations, which were scattered across the chart of nuclides. This scattering demonstrated that the applicability of the method was not limited to a specific area, though the results were generally more reliable with heavier isotopes. Many isotopes were also extrapolated by several mass relations, which provided several comparable estimates for the given isotopes. In addition, it allowed for a combined result based on extrapolations from different mass relations.

When comparing or combining different extrapolations some considerations had to be made. Some extrapolations had too large uncertainties and were excluded from any calculations. These extrapolations were considered too unreliable and would not improve the final result. If significant discrepancies were found when calculating the average, any extrapolation influenced by effects known to be significant was excluded as well.

Several extrapolations could be combined with these considerations in mind, and the final results were displayed in tables where several expected general tendencies were observed. In particular, results regarding heavier isotopes were more consistent and more reliable. The unique orientation of one mass relation along the stability curve resulted in fewer possible extrapolations comparable with the other three relations.

Comparisons of extrapolated results, based on any of the four mass relations, in general showed rather close agreement. Even when an extrapolation was discarded based on its uncertainty it was often in close vicinity to the final average. The extrapolations at higher nucleon number usually differed by at most $\sim 400 \mathrm{keV}$. These averages were consistent with other estimates, and the uncertainties were of the same magnitude. On the other hand, the averages seemed much less accurate for lighter isotopes, and the method is probably not competitive for these isotopes.

A number of the results in the region where $Z>82$ and $N<126$ were acceptable both in consistency and un- 
certainty. This is particularly interesting given that this region traditionally is very difficult to estimate. Some results, notably in this area, were influenced by shell effects, and should be handled with care, but generally the calculated averages seemed to be reliable.

Ten isotopes estimated by combining extrapolations were measured after Audi and Meng complied their initial information. This allowed for a direct evaluation of the accuracy of the method. The values for most extrapolations corresponded very well to the extrapolations. Those that deviated significantly were all in the vicinity of shells or subshells, and the inaccuracy of the method in such areas is not surprising, as no attempt has been made to account for these effects. On the contrary, it is more surprising that the extrapolation of ${ }^{138} \mathrm{I}$ is so accurate as this isotope is also close to shells.

The greatest fundamental weakness with the presented method is the use of somewhat removed isotopes. Isotopes are combined over a significant distance, particularly when calculating the variation in the tendencies. Combining isotopes over a greater distance increases the likelihood of combining unrelated effects. It is difficult to account for the effect of one isotope being influenced differently than the others in the extrapolation. The usual approach has been to apply more compact mass relations. Beginning with the Garvey-Kelson mass relations, this fear of combining unrelated effects has been an ongoing concern. However, despite the rather large span of the mass relations used here, the results are comparable to the best of other available extrapolations.

Although, applying other, possibly more compact, mass relations would be the most obvious way to supplement the results. By combining results from multiple mass relations, and not just the four applied here, this method also allows for convenient extensions and improvements. Applying more complex mass relations, could possibly increase the applicability of this method even more. By creating a comprehensive system of extrapolations based on different relations it would probably be possible to determine binding energies with still greater precision, and in greater number. The actual binding energy would be approached from many directions by several mass relations, and the final result would be all the more credible.

Even though the extrapolated binding energies might not be perfectly consistent, the calculated averages should still be very viable and useful estimates. In particular, results for isotopes unencumbered by shell effects and the like should be more than reliable.

Finally, from the evaluations of $Q$-values along the stability line it was possible to examine general structures in the binding energy of super-heavy isotopes. Here signature of a minor neutron shell at $N=152$ was found. Applying the mass relation perpendicular to the stability curve the behaviour across this neutron number was found to be characteristic of a closed shell. These findings very clearly suggests there exists a minor neutron shell at $N=152$. It is also striking that no other shell is revealed in this region which owes its very existence to stability provided by shell effects.

In conclusion, simple four-nucleus mass relations, where smooth contributions to the nuclear binding energy vanish to second order, are used to extrapolate unknown nuclear binding energies with rather good accuracy. We provide estimates for a series of different nuclei just outside the region of knowledge where a good deal of present nuclear research activities are focussed. In particular, we apply the method to the super-heavy region where special $Q$-values are measured very accurately.
[1] D. Lunney, J.M. Pearson and C. Thibault, Rev. Mod. Phys. 75 (2003) 1021

[2] M. Block et al., Nature 463 (2010) 785

[3] G. Audi, A.H. Wapstra and C. Thibaults, Nucl. Phys. A 729 (2003) 337

[4] A. Bohr and B.R. Mottelson, Nuclear Structure, Volume $I$ and II: Nuclear structure, World Scientific Pub. Co. (1998).

[5] W. D. Myers, Droplet of atomic nuclei (Plenum, NY, 1977)

[6] P. Möller, W.D. Myers, H. Sagawa and S. Yoshida, Phys. Rev. Lett. 108 (2012) 052501

[7] S. Goriely and J.M. Pearson, Phys. Rev. C77 (2008) 031301

[8] J. Erler, N. Birge, M. Kortelainen, W. Nazarewicz, E. Olsen, A.M. Perhac and M. Stoitsov, Nature 486 (2012) 509

[9] M. Brack and R.K. Bhaduri, Semiclassical Physics, (1997) (Frontiers in Physics), Perseus Books Group, ISBN: 0201483513.

[10] P. Navratil, S. Quaglione, I.Stetcu and B.R. Barrett, J.
Phys. G 36 (2009) 083101

[11] G.T. Garvey, W. J. Gerace, R. L. Jaffe and I. Talmi, Rev. Mod. Phys. 41 (1969) S1

[12] J. Mendoza-Temis, I. Morales, J. Bareab, A. Franka, J.G. Hirscha, J.C. Lopez Vieyra, P. Van Isacker, and V. Velazquez, Nucl. Phys. A812 (2008) 28

[13] J. Barea, A. Frank, J.G. Hirsch, P. Van Isacker, S. Pittel, and V. Velazquez, Phys. Rev. C77 (2008) 041304(R)

[14] J. Duflo, Nucl. Phys. A576 (1994), 29; J. Duflo and A.P. Zuker, Phys. Rev. C52 (1995) R23

[15] A. S. Jensen, P. G. Hansen, and B. Jonson, Nucl. Phys. A431 (1984) 393

[16] A. Molinari and H.A. Weidenmüller, Phys. Lett. B637 (2006) 48

[17] H. Olofsson, S. Åberg, O. Bohigas and P. Leboeuf, Phys. Rev. Lett. 96 (2006) 042502

[18] W.A. Friedman and G.F. Bertsch, Phys. Rev. C76 (2007) 057301

[19] K. Heyde and J.L. Wood, Rev. Mod. Phys. 83 (2011) 1467

[20] E. R. Minaya et al., Science 337 (2012) 1207 
[21] Georges Audi and Wang Meng, Private Communication April 2011.

[22] J. Hakala et al, Phys. Rev. Lett. 109 (2012) 032501

[23] J. Van Schelt et al, Phys. Rev. C85 (2012) 045805
[24] G. Audi, M. Wang, A.H. Wapstra, F.G. Kondev, M. MacCormick, X. Xu, and B. Pfeiffer, Chinese Phys. C36 (2012) 1287 